\title{
Uterine leiomyoma mimicking a gastrointestinal stromal tumor with chronic spontaneous hemorrhage: A case report
}

\author{
XIAOQING ZHU, JING FEI, WEIJIANG ZHANG and JIANWEI ZHOU \\ Department of Gynecology, Second Affiliated Hospital, School of Medicine, Zhejiang University, \\ Hangzhou, Zhejiang 310009, P.R. China
}

Received July 10, 2014; Accepted February 26, 2015

DOI: 10.3892/ol.2015.3083

\begin{abstract}
Uterine leiomyomas are the most common tumors to affect women. Cases of uterine leiomyoma mimicking a gastrointestinal stromal tumor with chronic spontaneous hemorrhage are extremely rare, and similar cases to that of the present study are yet to be reported. In the current study, a 48-year-old female presented with symptoms of lower abdominal pain after menses that had persisted for 4 months. The patient was initially diagnosed with uterine leiomyoma. However, according to the results of the gross and auxiliary examinations, a diagnosis of a gastrointestinal stromal tumor could not be excluded. Therefore, the patient underwent a rectal sigmoid bowel resection, subtotal hysterectomy and bilateral salpingectomy. Subsequent to surgery, the immunohistochemical examinations confirmed the initial diagnosis of uterine leiomyoma. The surgery had therefore involved an unnecessary organ resection.
\end{abstract}

\section{Introduction}

Uterine leiomyoma is the most common tumor to affect women. Fewer than 100 cases of massive hemoperitoneum resulting from the rupture of a uterine leiomyoma have been previously reported (1). However, a case of uterine leiomyoma mimicking a gastrointestinal stromal tumor (GIST) with chronic spontaneous hemorrhage is yet to be reported. Patients with uterine fibroids experience pelvic discomfort and excessive uterine bleeding (2). By contrast, patients with a GIST may not exhibit any typical clinical symptoms (3). In certain circumstances, uterine fibroids may mimic malignant tumors, particularly when examinations reveal that the fibroids have adhered to the bowels or mesentery (4). The fact that uterine leiomyomas occasionally mimic malignant tumors makes

Correspondence to: Dr Jianwei Zhou, Department of Gynecology, Second Affiliated Hospital, School of Medicine, Zhejiang University, 88 Jiefang road, Hangzhou, Zhejiang 310009, P.R. China

E-mail: jianwei-zhou@163.com

Key words: uterine fibroids, gastrointestinal stromal tumor, chronic spontaneous hemorrhage diagnosis challenging, and may result in misdiagnosis. The present study describes a case of uterine fibroid mimicking a GIST with chronic spontaneous hemorrhage that was initially misdiagnosed.

\section{Case report}

A 48-year-old female with regular menstruation was admitted to the Second Affiliated Hospital, School of Medicine, Zhejiang University (Hangzhou, China) with lower abdominal pain after menses that had persisted for 4 months. Evident tenderness of the uterus was identified upon physical examination. A transvaginal sonography detected multiple leiomyomas, the largest of which measured $1.5 \times 1.2 \mathrm{~cm}$. A hypoechoic mass measuring $6.5 \times 6.1 \mathrm{~cm}$ was also detected behind and to the left of the uterus. The mass was believed to be an old hematoma. In addition, pelvic effusion was observed. The patient was gravida 2, para 2, without a history of cesarean section. The patient had no history of taking oral contraceptives. However, 4 months prior to admission, the patient had experienced an ovarian corpus luteum rupture and received symptomatic treatment, including antibiotics, hemostasis and fluid infusion at The People's Hospital of Yuyao. Surgery was not performed.

Magnetic resonance imaging (MRI) revealed a mass occupying the fundus of the uterus. The mass was considered to be a tumor or subserous fibroid (Fig. 1). In addition, multiple leiomyomas and focal adenomyosis were detected. However, the indices of tumor markers, including cancer antigen (CA)-125, CA19-9 and carcinoembryonic antigen, were within normal limits. In addition, the indices of the complete blood count, urinalysis and electrolytes, as well as the liver enzyme studies, were all within normal limits.

The patient underwent a diagnostic laparoscopy. A gross examination revealed a pelvic mass measuring $6 \times 7 \mathrm{~cm}$, with chronic spontaneous hemorrhage. The mass was located between the sigmoid colon and the uterus, and was approximate to the rectum. The mass and the bowels exhibited ill-defined margins. The mass was white-tan in color with a whorl-like, trabeculated appearance; it was without a tumor pseudocapsule, and had encroached the mesentery and serosa of the sigmoid colon (Fig. 2). The appearance of the mass was similar to that of a malignant tumor. However, the appearance of the ovaries and fallopian tubes was normal. The mass was resected and sent for fast frozen section analysis. The pathological report 

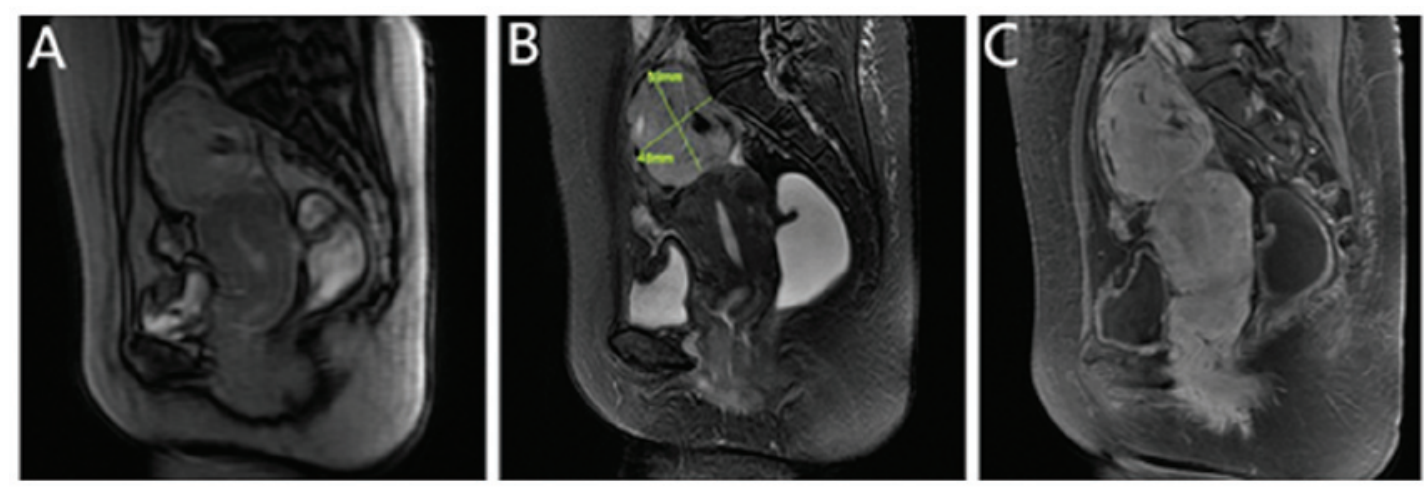

Figure 1. MRI images of the uterine leiomyoma. (A) T1-weighted MRI of the pelvis; (B) T2-weighted MRI of the pelvis; Green lines indicate the size and borders of the mass. (C) Contrast-enhanced MRI of the pelvis. MRI revealed a mass in the upper the fundus of the uterus, which demonstrated a long signal upon T1 and T2-weighted imaging. The signal was evidently enhanced upon contrast-enhanced MRI. MRI, magnetic resonance imaging.
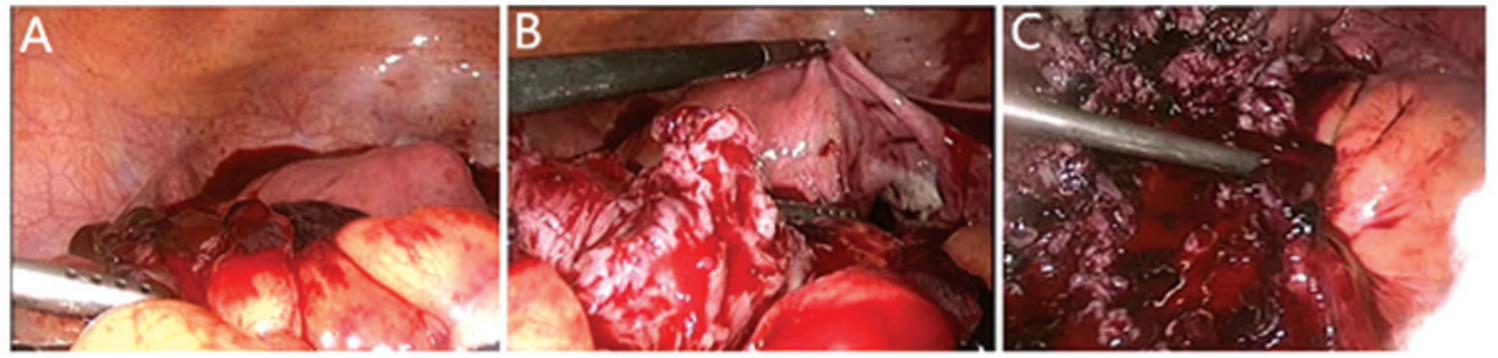

Figure 2. Laparoscopic images captured during surgery. (A) Image of the uterine leiomyoma prior to resection, revealing chronic spontaneous hemorrhage of the leiomyoma and pelvic hematocele. (B) Image of the pelvic mass during the resection, revealing that the uterine leiomyoma was white-tan in color with a whorl-like, trabeculated appearance. (C) Image of the pelvic mass post-partial resection of the uterine leiomyoma, revealing that the tumor exhibited an indistinct association with the colon.

of the fast frozen sections indicated a diagnosis of a spindle cell tumor. A GIST or leiomyoma were also considered. The pathologists suggested that the definitive diagnosis required further immunohistochemical examination. A consultation with the surgical oncology doctor established that a diagnosis of GIST could not be excluded. Therefore, to avoid the possibility of multiple surgeries, subsequent to obtaining informed consent, the patient underwent a rectal sigmoid bowel resection, subtotal hysterectomy and bilateral salpingectomy.

Following surgery, the patient was prescribed symptomatic treatment, including $2 \mathrm{~g}$ ceftizoxime via intravenous injection and $0.5 \mathrm{~g}$ ornidazole and sodium chloride twice a day to prevent infection from the operation incision. Additionally, the patient was in abrosia, therefore $2000 \mathrm{ml}$ glucose and sodium chloride were taken to provide the basic fluid requirement. A further pathological examination, including immunohistochemistry was performed. The morphological examination revealed that the tumor was connected to the uterus and mesentery. The cells exhibited mild cellular atypia with 2-3/10 high-power field nuclear fission. There was no evidence of malignancy, such as tumor necrosis or a high activity of nuclear fission (Fig. 3D). Immunohistochemically, the spindle cells were positive for smooth muscle actin (SMA), desmin and progesterone receptor (PR) (Fig. 3C), but negative for cluster of differentiation (CD)117 (Fig. 3B), CD34 (Fig. 3A), CD10, pan-cytokeratin, S100 protein, estrogen receptor and delay of germination 1 (DOG-1). Of these tumor markers, CD117, CD34 and DOG-1 are markers for GISTs. The clinical data and results of the immunohistochemistry indicated a diagnosis of uterine leiomyoma. However, the malignant potential of the tumor could not be validated. The pathologists advised that the patient should be given a follow-up examination. Although multiple fibroids were detected in the uterus, the fallopian tubes appeared normal.

As the malignant potential of the tumor was uncertain, a consultation with the University of California at Los Angeles (UCLA) was arranged. Pathologists at the UCLA suggested that the immunohistochemical phenotype and morphological features of the mass favored the diagnosis of a benign uterine leiomyoma. The mass was positive for the smooth muscle markers, desmin and SMA, but negative for the GIST markers, CD117, CD34 and DOG-1. In the sections of the tumor that were analyzed, there was no evidence of malignancy, such as tumor necrosis or a high mitotic activity. The positive staining for PR was consistent with a uterine origin, and given the patient's history, this may have represented an exophytic leiomyomatous growth from the uterine surface. The combination of the clinical impression and the results of the immunohistochemical examination established a final diagnosis of multiple uterine leiomyomas.

\section{Discussion}

Uterine leiomyoma is one of the most common benign gynecological tumors. Leiomyomas appear grossly as round, well-circumscribed, but not encapsulated, solid nodules with pseudocapsules that are white or tan, and exhibit a whorled 


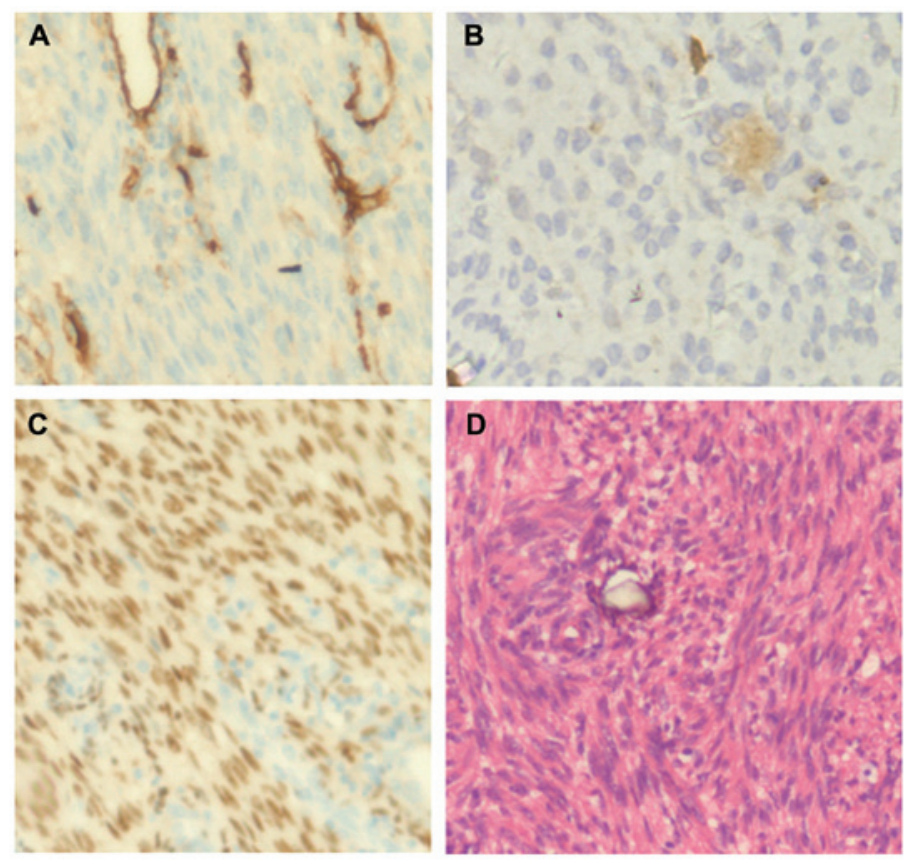

Figure 3. Histological features and immunohistochemical results of the uterine leiomyoma. The tumor cells were negative for (A) CD34 and (B) CD117, but demonstrated marked positivity for (C) progesterone receptor (immunostaining; magnification, x400). (D) The morphological examination did not reveal tumor necrosis or a high activity of nuclear fission (hematoxylin and eosin staining; magnification, x400). CD, cluster of differentiation.

appearance upon histological analysis. Leiomyomas may disrupt the functions of the uterus and result in excessive uterine bleeding, anemia, defective implantation of an embryo, recurrent pregnancy loss, preterm labor, obstruction of labor, pelvic discomfort and urinary incontinence. In addition, they may mimic or conceal malignant lesions (5). The gold standard diagnostic approach for uterine fibroids is gray-scale ultrasonography, followed by MRI for complex clinical cases (6).

GISTs are the most common mesenchymal tumors of the gastrointestinal tract. GISTs appear grossly as grayish-white or red, nodular or lobulated, solid nodules without a pseudocapsule. GISTs are characterized by the expression of KIT (also known as CD117) and DOG-1, which was identified by transcriptome analysis (7). GISTs often lack typical clinical manifestations and are usually incidentally detected in the small intestinal or gastric serosa during surgery for other conditions. GISTs exhibit two morphological variants; the common spindle cell subtype and the epithelioid subtype (8). A large number of the individual spindle-shaped cells, which are scattered throughout the smear background, contain bland nuclei and a high nuclear cytoplasmic ratio. The spindle cell subtype of GISTs has nuclei that are elongated with blunt ends. Necrosis is rarely, if ever, observed (8). In the present study, the morphological features of the tumor simulated the features of a GIST.

The differential diagnosis between a uterine leiomyoma and a GIST has been referred to previously (3). However, cases of uterine leiomyoma mimicking GISTs have not yet been reported. Furthermore, no guidelines exist concerning recommended procedures for differential diagnosis or appropriate therapies. In the present study, gross examination identified a tumor without a pseudocapsule that had encroached upon the mesentery and serosa of the sigmoid colon. The appearance simulated that of a GIST. In addition, the report on the surgical frozen sections indicated a spindle cell tumor, which simulated the morphology of a GIST. The condition of the uterine leiomyoma mimicking a GIST lead to an initial misdiagnosis. It is therefore important for clinicians to be aware of the differential diagnosis for GISTs and uterine leiomyomas.

Prognostic factors for GISTs are the mitotic rate, tumor size and tumor site. It has been established that gastric GISTs exhibit improved prognoses compared with small bowel or rectal GISTs. The surgical margins and the presence/absence of tumor rupture are additional factors that affect prognosis (9). In accordance with the European Society for Medical Oncology clinical practice guidelines, the patient initially underwent a rectal sigmoid bowel resection, subtotal hysterectomy and bilateral salpingectomy following the misdiagnosis. According to the guidelines, when GIST nodules $<2 \mathrm{~cm}$ in size are detected, laparoscopic or laparotomic excision may be the only way to establish a histological diagnosis. The standard approach for nodules measuring $\geq 2 \mathrm{~cm}$ in size, however, is biopsy or excision. If a mass is detected, particularly if surgery is likely to consist of a multivisceral resection, the standard approach is to perform multiple core needle biopsies (9).

As a result of the misdiagnosis in the present case, we suggest that a biopsy of the pelvic mass should be performed in cases where a condition of uterine leiomyoma mimics a GIST. When examinations prior to surgery identify a pelvic mass with pelvic effusion, a diagnosis of leiomyoma with spontaneous hemorrhage should be considered. In cases where clinicians identify a pelvic mass with spontaneous hemorrhage, and a diagnosis cannot be verified by the pathological examination of the surgical fast sections, removal of the pelvic mass should be performed first. Further therapy can be decided upon according to the results of a second pathological examination. This should avoid unnecessary resection of organs. In addition, if required, gastroscopy and colonoscopy can be performed in order to exclude the diagnosis of GISTs prior to laparoscopy. 
Cases of acute spontaneous hemorrhage of uterine leiomyomas have been previously reported. However, this is a rare complication of a uterine leiomyoma (2). According to the literature, acute spontaneous hemorrhage of a uterine leiomyoma results from the spontaneous rupture of veins or arteries $(10,11)$. Patients are admitted to an emergency department with the acute onset of abdominal pain or hypovolemic shock (12). In the present study, the uterine leiomyoma was accompanied by chronic spontaneous hemorrhage. Similar cases are yet to be reported. The mechanisms underlying the spontaneous hemorrhage of a uterine leiomyoma are yet to be elucidated. However, several have been proposed. The most putative mechanism is that increasing abdominal pressure leads to spontaneous hemorrhage. It has been hypothesized that increased abdominal pressure could result in the passive congestion and rupture of the superficial veins (8). However, Grisaru et al (13) revealed that degeneration of a uterine leiomyoma can result in spontaneous perforation, which may also lead to hemorrhage.

The aim of the present study was to increase awareness among clinicians. GISTs should be considered during the differential diagnosis of uterine leiomyomas. Furthermore, when sonography identifies a uterine leiomyoma with pelvic effusion, spontaneous hemorrhage of the uterine leiomyoma should be taken into consideration. When a uterine leiomyoma mimics a GIST, further evaluation should be conducted in order to verify the diagnosis. If the diagnosis of GIST cannot be excluded, surgical resection of the mass can be performed first.

\section{Acknowledgements}

The authors would like to thank the patient and all individuals associated with present study.

\section{References}

1. Estrade-Huchon S, Bouhanna P, Limot O, et al: Severe life-threatening hemoperitoneum from posttraumatic avulsion of a pedunculated uterine leiomyoma. J Minim Invasive Gynecol 17: 651-652, 2010.

2. Gupta S: Acute complications of fibroids. Best Prac Res Clin Obstet Gynaecol 23: 609-617, 2009.

3. Miettinen M and Lasota J: Gastrointestinal stromal tumors - definition, clinical, histological,immunohistochemical and molecular genetic features and differential diagnosis. Virchows Arch 438: $1-12,2001$.

4. Abraham SC. Distinguishing gastrointestinal stromal tumors from their mimics: an update. Advances In Anatomic Pathology. 2007 14: 178-188.

5. Bulun SE: Uterine fibroids. N Engl J Med 369: 1344-1355, 2013.

6. Khan AT, Shehmar M and Gupta JK: Uterine fibroids: current perspectives. Int J Womens Health 6: 95-114, 2014.

7. Emile JF: Histology and molecular biology of GIST. Bull Acad Natl Med 196: 835-844, 2012 (In French).

8. Layfield LJ and Wallander ML: Diagnosis of gastrointestinal stromal tumors from minute specimens: cytomorphology, immunohistochemistry and molecular diagnostic findings. Diagn Cytopathol 40: 484-490, 2012.

9. ESMO/European Sarcoma Network Working Group: Gastrointestinal stromal tumors: ESMO clinical practice guidelines for diagnosis, treatment and follow-up. Ann Oncol 23 (Suppl 7): vii49-vii55, 2012.

10. Ymele FF, Tsuala JF, Fouedjio JH, Nangué C, De Kayo CK, Dobgima PW and Mbu RE: A rare complication of uterine leiomyomas: massive hemoperitoneum secondary to ruptured varices. Pan Afr Med J 14: 110, 2013 (In French).

11. Horowitz E, Dekel A, Feldberg D and Rabinerson D: Massive hemoperitoneum due to rupture of an artery overlying a uterine leiomyoma: a case report. Acta Obstet Gynecol Scand 84: 408-409, 2005.

12. Lotterman S: Massive hemoperitoneum resulting from spontaneous rupture of uterine leiomyoma. Am J Emerg Med 26: 971.e1-974.e2, 2008.

13. Grisaru D, Bar-Am A and Lessing JB: Spontaneous perforation of a degenerative nongestational uterine leiomyoma. Obstet Gynecol 87: 882, 1996. 